MATHEMATICS OF COMPUTATION

Volume 72 , Number 241, Pages 289-305

S 0025-5718(01)01407-7

Article electronically published on December 5,2001

\title{
THE CONVERGENCE OF SHOOTING METHODS FOR SINGULAR BOUNDARY VALUE PROBLEMS
}

\author{
OTHMAR KOCH AND EWA B. WEINMÜLLER
}

\begin{abstract}
We investigate the convergence properties of single and multiple shooting when applied to singular boundary value problems. Particular attention is paid to the well-posedness of the process. It is shown that boundary value problems can be solved efficiently when a high order integrator for the associated singular initial value problems is available. Moreover, convergence results for a perturbed Newton iteration are discussed.
\end{abstract}

\section{Preliminaries}

We discuss the numerical solution of the following nonlinear singular boundary value problems of the first order:

$$
\begin{aligned}
& y^{\prime}(t)=\frac{M(t)}{t} y(t)+f(t, y(t)), \quad t \in(0,1], \\
& g(y(0), y(1))=0, \\
& y \in C[0,1],
\end{aligned}
$$

where $y$ and $f$ are vector-valued functions of dimension $n, M$ is an $n \times n$ matrix and $g: \mathbb{R}^{n} \times \mathbb{R}^{n} \rightarrow \mathbb{R}^{r}, r \leq n$, is a smooth function.

The search for a method to solve problems (1.1) is strongly motivated by numerous applications from physics (see [3], [5], [6]), chemistry (cf. 30]) and mechanics (buckling of spherical shells, [4], 7]), as well as research activities in related areas (8], [26, 27], 28]).

Our aim is to investigate the convergence of shooting procedures (see [1] or [18]) for the approximate solution of (1.1), based on an efficient numerical solution of the associated singular initial value problems.

The unsatisfactory convergence properties of direct higher order methods are the main motivation to use shooting methods for the solution of singular boundary value problems. For collocation schemes only the stage order can be observed; the superconvergence does not hold in general, see [16]. In the presence of a singularity, other direct higher order methods (finite differences) show order reductions and become inefficient. Moreover, the standard acceleration techniques based on low-order methods do not work efficiently either, because in general, a proper asymptotic error expansion for the basic scheme does not exist, cf. [12]. Moreover, multiple shooting seems to be a particularly attractive alternative, because within its framework one can use different controlling mechanisms close to and away from

Received by the editor February 10, 2000 and, in revised form, January 3, 2001.

2000 Mathematics Subject Classification. Primary 65L10.

This project was supported by the Austrian Research Fund (FWF) grant P-12507-MAT.

(C)2001 American Mathematical Society 
the singular point. In the context of initial value problems, we have already gained some information on how the usual strategies for error estimation and grid selection work when they are applied to singular problems. These observations suggest that we will possibly need to control the global error close to the singularity and switch to the control of the local error away from the singular point. This idea can be easily realized in a code based on the multiple shooting approach.

For the solution of initial value problems, various standard methods have been investigated. It turns out, however, that numerical methods widely used for the solution of regular initial value problems show order reductions in the singular case. See [15] for a discussion of multistep methods, and [17] for explicit Runge-Kutta schemes. Therefore, we use Iterated Defect Correction (IDeC) based on the implicit Euler method for the solution of the involved initial value problems. This acceleration technique was investigated for regular problems in [9], [10 and 11. When applied to singular initial value problems, this method shows its classical convergence behavior, which means that any convergence order $O\left(h^{p}\right)$ can be obtained comparatively cheaply for sufficiently smooth data; see [22 for the analysis and [2], 21] for numerical evidence. Throughout this paper we will therefore assume that a high-order method for the solution of initial value problems is available (without referring to any details of the procedure).

The analytical properties of the boundary value problems (1.1) have been discussed in full detail in [14]. The analysis of the related initial value problems has been given in [20] and [21. In Section 2 we briefly recapitulate analytical results important for the subsequent discussion of the numerical methods. Single shooting is studied for the linear case and for the nonlinear case in Section 3 and Section 4 respectively. In Section 5 the convergence of multiple shooting and Newton's iteration is considered. Numerical experiments illustrating the theory are discussed in Section 6.

In the sequel, the following notation is used. We denote by $\mathbb{C}^{n}$ the space of complex-valued vectors of dimension $n$ and use $|\cdot|$ to denote the maximum norm in $\mathbb{C}^{n}$,

$$
|x|=\left|\left(x_{1}, x_{2}, \cdots, x_{n}\right)^{T}\right|:=\max _{1 \leq i \leq n}\left|x_{i}\right| .
$$

$C_{n}^{p}[0,1]$ is the space of complex vector-valued functions which are $p$ times continuously differentiable on $[0,1]$. For every function $y \in C_{n}^{0}[0,1]$ we define the maximum norm,

$$
\|y\|:=\max _{0 \leq t \leq 1}|y(t)| .
$$

We will also use the maximum norm restricted to the interval $[0, \delta], 0<\delta \leq 1$,

$$
\|y\|_{\delta}:=\max _{0 \leq t \leq \delta}|y(t)| .
$$

$C_{n \times n}^{p}[0,1]$ is the space of complex-valued $n \times n$ matrices with columns in $C_{n}^{p}[0,1]$. For a matrix $A=\left(a_{i j}\right)_{i, j=1}^{n}, A \in C_{n \times n}^{0}[0,1],\|A\|$ is the induced norm,

$$
\|A\|:=\max _{0 \leq t \leq 1}|A(t)|=\max _{0 \leq t \leq 1}\left(\max _{1 \leq i \leq n} \sum_{j=1}^{n}\left|a_{i j}(t)\right|\right),
$$

and $\|A\|_{\delta}$ is defined in an obvious way. Where there is no confusion we will omit the subscripts $n$ and $n \times n$ and write $C=C[0,1]=C^{0}[0,1]$. 
For a constant matrix $A$ the kernel of $A$ is denoted by $\operatorname{ker}(A)$. Throughout, we write $I_{n}$ for the identity matrix in $\mathbb{R}^{n \times n}$. Moreover, $\operatorname{span}(A)$ denotes the closed hull of the columns of $A$, where $A$ is an arbitrary matrix.

For the numerical analysis, we define equidistant grids of the form

$$
\Delta_{h}:=\left(t_{0}, t_{1}, \ldots, t_{N}\right)
$$

where $t_{i}=i h, i=0, \ldots, N, h=\frac{1}{N}$, and grid vectors

$$
u_{h}:=\left(u_{0}, \ldots, u_{N}\right) \in \mathbb{C}^{(N+1) n} .
$$

The norm on the space of grid vectors is defined as

$$
\left\|u_{h}\right\|_{h}:=\max _{0 \leq k \leq N}\left|u_{k}\right| \text {. }
$$

For a continuous function $y \in C[0,1]$, we denote by $R_{h}$ the projection onto the space of grid vectors,

$$
R_{h}(y):=\left(y\left(t_{0}\right), \ldots, y\left(t_{N}\right)\right) .
$$

\section{ANALYSis OF SINGULAR BOUNDARY AND INITIAL VALUE PROBLEMS}

In this section we consider the singular boundary value problem (1.1). The analytical properties of (1.1) have been studied in [14]. Here, we assume all eigenvalues of $M(0)$ to have nonpositive real parts. Moreover, the only eigenvalue of $M(0)$ on the imaginary axis is zero. These restrictions are necessary to ensure the well-posedness of the initial value problems associated with (1.1).

First, we treat the linear case,

$$
\begin{aligned}
& y^{\prime}(t)=\frac{M(t)}{t} y(t)+f(t), \quad t \in(0,1], \\
& B_{a 2} y(0)+B_{b 2} y(1)=\beta_{2}, \\
& y \in C[0,1],
\end{aligned}
$$

where $B_{a 2}, B_{b 2} \in \mathbb{R}^{r \times n}, r<n$, are constant matrices, and $\beta_{2} \in \mathbb{R}^{r}$ is a constant vector.

Throughout, we assume $M \in C^{1}[0,1]$. Equivalently, we can rewrite $M(t)$ and obtain

$$
M(t)=M(0)+t C(t)
$$

with a continuous matrix $C(t)$.

Let $X_{0}$ be the eigenspace of $M(0)$ corresponding to the eigenvalue $\lambda=0$ and let $R$ be a projection 1$]$ onto $X_{0}$. We define

$$
S:=I_{n}-R
$$

where we denote by $I_{n}$ the $n \times n$ identity matrix. The necessary and sufficient condition for $y$ to be continuous on $[0,1]$ is

$$
S y(0)=0 .
$$

This yields

$$
y(0)=(S+R) y(0)=R y(0),
$$

and, because

$$
M(0) y(0)=M R y(0)=0,
$$

\footnotetext{
${ }^{1} R$ is called spectral projection.
} 
it follows that $(2.1 \mathrm{C})$ is equivalent to $y \in \operatorname{ker}(M(0))$. This can also be expressed as

$$
B_{a 1} y(0)=0,
$$

where $B_{a 1} \in \mathbb{R}^{(n-r) \times n}$ consists of the linearly independent rows of $M(0)$. These conditions are augmented by (2.1b) to yield a unique solution, see below.

We denote by $\tilde{E}$ the $n \times r$ matrix consisting of the linearly independent columns of $R$. Moreover, let $\bar{\Phi}(t)$ be the fundamental solution matrix of the initial value problem

$$
\begin{aligned}
& \bar{\Phi}^{\prime}(t)=\frac{M(t)}{t} \bar{\Phi}(t), \quad t \in(0,1], \\
& \bar{\Phi}(0)=\tilde{E} .
\end{aligned}
$$

Consequently, the necessary and sufficient condition for the problem (2.1) to have a unique solution is that the $r \times r$ matrix $\bar{Q}$,

$$
\bar{Q}:=B_{a 2} \tilde{E}+B_{b 2} \bar{\Phi}(1),
$$

is nonsingular. If $f$ is $k$ times continuously differentiable and $M \in C^{k+1}[0,1]$, then $y \in C^{k+1}[0,1]$.

For the nonlinear problem 2

$$
\begin{aligned}
& y^{\prime}(t)=\frac{M(t)}{t} y(t)+f(t, y(t)), \quad t \in(0,1], \\
& g(y(0), y(1))=0, \\
& y \in C[0,1],
\end{aligned}
$$

the analogous smoothness properties can be shown. Let us make the following assumptions:

1. $f: D_{1} \rightarrow \mathbb{R}^{n}$ and $g: D_{2} \rightarrow \mathbb{R}^{m}$ are nonlinear mappings, where $D_{1} \subseteq[0,1] \times \mathbb{R}^{n}$ and $D_{2} \subseteq \mathbb{R}^{n} \times \mathbb{R}^{n}$ are suitable sets. Motivated by the linear case, we choose $m=r$.

2. Equation (2.5) has a solution $y \in C[0,1] \cap C^{1}(0,1]$. With this solution and a $\rho>0$ we associate the sphere

$$
S_{\rho}(y(t)):=\left\{z \in \mathbb{R}^{n}:|y(t)-z| \leq \rho\right\}
$$

and the tube

$$
T_{\rho}:=\left\{(t, z): t \in[0,1], z \in S_{\rho}(y(t))\right\} .
$$

3. $f(t, z)$ is continuously differentiable with respect to $z$, and $\frac{\partial f(t, z)}{\partial z}$ is continuous on $T_{\rho}$. Also, $g(v, w)$ is continuously differentiable on $S_{\rho}(y(0)) \times S_{\rho}(y(1))$.

4. The solution $y(t)$ is isolated. This means that

$$
\begin{aligned}
& u^{\prime}(t)=\frac{M(t)}{t} u(t)+A(t) u(t), \quad t \in(0,1], \\
& B_{a 2} u(0)+B_{b 2} u(1)=0, \\
& u \in C[0,1],
\end{aligned}
$$

\footnotetext{
${ }^{2}$ Again, $M(0)$ has only eigenvalues with negative real parts or the eigenvalue 0 .
} 
where

$$
\begin{aligned}
& A(t):=\frac{\partial f(t, z)}{\partial z}(t, y(t)), \\
& B_{a 2}:=\frac{\partial g(v, w)}{\partial v}(y(0), y(1)), \quad B_{b 2}:=\frac{\partial g(v, w)}{\partial w}(y(0), y(1)),
\end{aligned}
$$

has only the trivial solution.

Under these assumptions and for $f \in C^{k}\left(T_{\rho}\right), M \in C^{k+1}[0,1]$, the solution $y$ of (2.5) satisfies $y \in C^{k+1}[0,1]$.

For further details and proofs, see [14].

As a special case of a boundary value problem we now consider the following singular initial value problem:

$$
\begin{aligned}
& z^{\prime}(t)=\frac{M(t)}{t} z(t)+f(t, z(t)), \quad t \in(0,1], \\
& B_{a 2} z(0)=\beta_{2}, \\
& z \in C[0,1],
\end{aligned}
$$

where $z, f$ are vector-valued functions of dimension $n, M \in C^{1}[0,1], B_{a 2}$ is an $r \times n$ matrix and $\beta_{2}$ is a vector of dimension $r \leq n$.

The analytical properties of (2.6) have been investigated in full detail in [20] and [21. In this analysis we assume that $f(t, z)$ is continuous and satisfies a Lipschitz condition with respect to $z$ on $[0,1] \times \mathbb{R}^{n}$. We require that $M(0)$ has no purely imaginary eigenvalues or eigenvalues with positive real parts, and show that this restriction is necessary for (2.6) to be well-posed. Moreover, the condition $M(0) z(0)=0$ is necessary and sufficient for $z \in C[0,1]$. Also, the solution of (2.6) is unique iff the matrix $B_{a 2} \tilde{E}$ is nonsingular. If $f \in C^{k}\left([0,1] \times \mathbb{R}^{n}\right)$ and $M \in C^{k+1}[0,1]$, the solution satisfies $\left.z \in C^{k+1}[0,1]\right]^{3}$

\section{Single SHOOTING-LINEAR PROBLEMS}

We first consider the linear boundary value problem

$$
\begin{aligned}
& y^{\prime}(t)=\frac{M(t)}{t} y(t)+f(t), \quad t \in(0,1] \\
& \left(\begin{array}{c}
B_{a 1} \\
B_{a 2}
\end{array}\right) y(0)+\left(\begin{array}{c}
0 \\
B_{b 2}
\end{array}\right) y(1)=\left(\begin{array}{c}
0 \\
\beta_{2}
\end{array}\right),
\end{aligned}
$$

where $B_{a 1} \in \mathbb{R}^{(n-r) \times n}$ consists of the linearly independent rows of $M(0)$. As mentioned in Section 2 , we assume that all eigenvalues of $M(0)$ have negative real parts or are equal to zero. Moreover, we assume the above boundary value problem to be well-posed. The necessary condition for the problem to be well-posed can be expressed as $M(0) y(0)=0$, and this restriction is reflected in the special form of the partially separated boundary conditions (3.1b). Also the matrix $\bar{Q}$ from (2.4) has to be nonsingular. The exact solution of the boundary value problem (3.1) solves the equivalent initial value problem

$$
\begin{aligned}
& z^{\prime}(t)=\frac{M(t)}{t} z(t)+f(t), \quad t \in(0,1], \\
& z(0)=z_{0},
\end{aligned}
$$

\footnotetext{
${ }^{3}$ Most of these results are direct consequences of the analysis for (2.5). It should be stressed, however, that under the above assumptions, even the existence of a unique solution of the nonlinear initial value problem can be shown.
} 
where $z_{0}$ satisfies

$$
z_{0}=\bar{\Phi}(0) \bar{s}+v(0)=\tilde{E} \bar{s}
$$

and the parameter vector $\bar{s} \in \mathbb{R}^{r}$ is suitably chosen. The matrices $\bar{\Phi}$ and $\tilde{E}$ are specified in (2.3), and $v$ is the solution of the inhomogeneous initial value problem,

$$
\begin{aligned}
& v^{\prime}(t)=\frac{M(t)}{t} v(t)+f(t), \quad t \in(0,1], \\
& v(0)=0 .
\end{aligned}
$$

The parameter vector $\bar{s}$ can be computed from the system

$$
\bar{Q} \bar{s}=\bar{\beta},
$$

where $\bar{Q}$ is given in (2.4) and

$$
\bar{\beta}=\beta_{2}-B_{b 2} v(1) .
$$

Note that (3.4) has a unique solution iff the boundary value problem is well-posed.

This approach to computing a shooting parameter $\bar{s} \in \mathbb{R}^{r}$ with $r<n$ is made possible by the special form of the boundary conditions $(3.1 \mathrm{~b})$. For the singular problem (3.1a), the restriction to starting vectors $z_{0} \in \operatorname{span}(\tilde{E})=\operatorname{ker}(M(0))$ is even necessary to obtain a well-posed initial value problem. This method, especially designed for problems with partially separated boundary conditions, is called reduced superposition or the method of complementary functions, cf. [1, [19].

In order to solve (3.1) we choose an equidistant grid $\Delta_{h}$, and solve all initial value problems involved in the algorithm described above by applying a solution method with level of accuracy $O\left(h^{p}\right)$ to obtain numerical approximations $\bar{Q}_{h}$ and $\bar{\beta}_{h}$ for $\bar{Q}$ and $\bar{\beta}$. The numerical approximation $\bar{s}_{h}$ for $\bar{s}$ is then determined from the perturbed system of linear equations

$$
\bar{Q}_{h} \bar{s}_{h}=(\bar{Q}+E) \bar{s}_{h}=\bar{\beta}_{h}=\bar{\beta}+e,
$$

where

$$
|E|,|e|=O\left(h^{p}\right) .
$$

It can easily be shown using Banach's Lemma that

$$
\left|\bar{s}_{h}-\bar{s}\right|=O\left(h^{p}\right)
$$

if we choose $h$ in such a way that $\left|\bar{Q}^{-1} E\right|<1$. Finally, the solution $\tilde{z}_{h}$ on the grid $\Delta_{h}$ is determined by solving the perturbed initial value problem

$$
\begin{aligned}
& \tilde{z}^{\prime}(t)=\frac{M(t)}{t} \tilde{z}(t)+f(t), \quad t \in(0,1], \\
& \tilde{z}(0)=z_{h, 0}:=\tilde{E} \bar{s}_{h} .
\end{aligned}
$$

The error function

$$
\varepsilon(t)=z(t)-\tilde{z}(t)
$$

satisfies the homogeneous initial value problem

$$
\begin{aligned}
& \varepsilon^{\prime}(t)=\frac{M(t)}{t} \varepsilon(t), \quad t \in(0,1], \\
& \varepsilon(0)=\varepsilon_{0}:=z(0)-\tilde{z}(0),
\end{aligned}
$$


and equivalently, it satisfies the integral equation (the matrix $C(t)$ is given via $(2.2)$ )

$$
\varepsilon(t)=\varepsilon_{0}+t \int_{0}^{1} \tau^{-M(0)} C(\tau t) \varepsilon(\tau t) d \tau,
$$

see [20] and [21]. For a sufficiently small $\delta>0$ this yields

$$
\|\varepsilon\|_{\delta} \leq \text { const. }\left|\varepsilon_{0}\right| \leq \text { const. }|\tilde{E}|\left|\bar{s}_{h}-\bar{s}\right|=O\left(h^{p}\right) .
$$

Since (3.6a) is a regular problem on $[\delta, 1]$, it follows from classical theory that the same result holds on the entire interval $[0,1]$.

The problem (3.5) is well-posed and can be solved in a stable way. This means that its numerical solution $\tilde{z}_{h}$ satisfies

$$
\left\|\tilde{z}_{h}-R_{h}(\tilde{z})\right\|_{h}=O\left(h^{p}\right),
$$

and finally,

$$
\left\|\tilde{z}_{h}-R_{h}(z)\right\|_{h}=\left\|\tilde{z}_{h}-R_{h}(y)\right\|_{h}=O\left(h^{p}\right) .
$$

Consequently, the approximation for the solution of the singular boundary value problem obtained by the shooting method converges with the order of accuracy of the solver used for the approximation of the underlying initial value problem.

\section{Single SHOOTING-NONLINEAR PROBLEMS}

Now we investigate the nonlinear boundary value problem

$$
\begin{aligned}
& y^{\prime}(t)=\frac{M(t)}{t} y(t)+f(t, y(t)), \quad t \in(0,1], \\
& B_{a 1} y(0)=0, \\
& g(y(0), y(1))=0,
\end{aligned}
$$

where $B_{a 1} \in \mathbb{R}^{(n-r) \times n}$ consists of the linearly independent rows of $M(0)$, and $g(v, w)$ is a smooth $r$-dimensional function. We assume that there exists an isolated solution $y \in C^{1}[0,1]$ of (4.1), and that the only eigenvalue of $M(0)$ with nonnegative real part is zero.

The boundary value problem (4.1) is equivalent to the initial value problem

$$
\begin{aligned}
& z_{\bar{s}}^{\prime}(t)=\frac{M(t)}{t} z_{\bar{s}}(t)+f\left(t, z_{\bar{s}}(t)\right), \quad t \in(0,1], \\
& z_{\bar{s}}(0)=\tilde{E} \bar{s},
\end{aligned}
$$

where $\bar{s}$ is determined from the nonlinear system of equations

$$
F(\bar{s}):=g\left(\tilde{E} \bar{s}, z_{\bar{s}}(1)\right)=0 .
$$

Again, the choice $z_{\bar{s}}(0)=\tilde{E} \bar{s}$, where $\tilde{E}$ is a basis of the kernel of $M(0)$, is equivalent to the condition (4.1b).

We solve (4.3) using Newton's method. For regular boundary value problems, it has been shown in [32] that the Newton iteration is well-defined and converges quadratically, provided that its starting value is sufficiently close to $s^{*}$ satisfying $y(0)=\tilde{E} s^{*}$. We now extend this result to singular problems and to the perturbed Newton iteration resulting from the numerical solution of the involved initial value problems. 
For a suitable initial guess $\bar{s}^{0}$ the Newton iteration for (4.3) is given by

$$
A\left(\bar{s}^{m}\right)\left(\bar{s}^{m+1}-\bar{s}^{m}\right)=-g\left(z_{\bar{s}^{m}}(0), z_{\bar{s}^{m}}(1)\right), \quad m=0,1, \ldots,
$$

where

$$
\begin{aligned}
A\left(\bar{s}^{m}\right) & =\frac{\partial}{\partial \bar{s}^{m}} g\left(z_{\bar{s}^{m}}(0), z_{\bar{s}^{m}}(1)\right) \\
& =\frac{\partial g(v, w)}{\partial v}\left(z_{\bar{s}^{m}}(0), z_{\bar{s}^{m}}(1)\right) \tilde{E}+\frac{\partial g(v, w)}{\partial w}\left(z_{\bar{s}^{m}}(0), z_{\bar{s}^{m}}(1)\right) \frac{\partial z_{\bar{s}^{m}}(1)}{\partial \bar{s}^{m}} .
\end{aligned}
$$

If $z_{\bar{s}^{m}}(t)$ is differentiable with respect to $\bar{s}^{m}, x(t)=\frac{\partial z_{\bar{s}} m(t)}{\partial \bar{s}^{m}}$ can be computed by solving the matrix initial value problem

$$
\begin{aligned}
& x^{\prime}(t)=\frac{M(t)}{t} x(t)+\frac{\partial f(t, z)}{\partial z}\left(t, z_{\bar{s}^{m}}(t)\right) x(t), \quad t \in(0,1], \\
& x(0)=\tilde{E} .
\end{aligned}
$$

Now we show that for a smooth function $f(t, z), z_{\bar{s}}$ depends smoothly on $\bar{s}$.

Lemma 4.1. If $f(t, z)$ is continuous on $[0,1] \times \mathbb{R}^{n}$ and two times continuously differentiable with respect to $z$ with bounded derivatives, then the solution $z_{\bar{s}}$ of (4.2) is two times continuously differentiable with respect to $\bar{s}$.

Proof. It follows from [20] and [21] that $x$ from (4.5) satisfies the integral equation

$$
\begin{aligned}
x(t)= & \tilde{E}+t \int_{0}^{1} \tau^{-M(0)} \frac{\partial f(t, z)}{\partial z}\left(\tau t, z_{\bar{s}}(\tau t)\right) x(\tau t) d \tau \\
& +t \int_{0}^{1} \tau^{-M(0)} C(\tau t) x(\tau t) d \tau .
\end{aligned}
$$

For a sufficiently small $\delta_{1}$ this equation has a unique continuous (with respect to $t$ ) solution in $\left[0, \delta_{1}\right]$. The above equation is equivalent to

$$
\left(I_{n}-K\right) x(t)=\tilde{E}
$$

where

$$
(K x)(t):=t \int_{0}^{1} \tau^{-M(0)} \frac{\partial f(t, z)}{\partial z}\left(\tau t, z_{\bar{s}}(\tau t)\right) x(\tau t) d \tau+t \int_{0}^{1} \tau^{-M(0)} C(\tau t) x(\tau t) d \tau
$$

is contracting on $\left[0, \delta_{1}\right]$. Consequently, the mapping $s \mapsto x(t) s$ is linear and bounded for all $t \in\left[0, \delta_{1}\right]$.

For the second derivative, we formally differentiate the above integral equation and show that the resulting equation has a continuous solution (a bounded bilinear mapping) on an interval $\left[0, \delta_{2}\right]$. Thus, the statement follows on $[0, \delta], \delta:=$ $\min \left\{\delta_{1}, \delta_{2}\right\}$. On the interval $[\delta, 1]$, classical theory yields the result, cf. [33].

Remark. We assumed that the boundary value problem (4.1) has an isolated solution and therefore it would be sufficient to require the smoothness and boundedness properties of $f(t, z)$ to hold merely on a tube $[0,1] \times\left\{y \in \mathbb{R}^{n}:|y| \leq R\right\}$ with a sufficiently large $R$. This can be easily seen from estimates derived in [20] and 21]. For reasons of simplicity, however, we will throughout the paper require the assumptions to hold on the whole domain $[0,1] \times \mathbb{R}^{n}$.

In order to show that the Newton iteration (4.4) is well-defined and converges quadratically in a neighborhood of the isolated solution, we only have to prove that the matrix $A\left(\bar{s}^{*}\right)$ is nonsingular at $\bar{s}^{*}$ associated with the isolated solution $z$, 
$z(0)=\tilde{E} \bar{s}^{*}$. This follows immediately from [14]. We formulate this result in the next theorem.

Theorem 4.2. Let $f(t, z)$ be continuous on $[0,1] \times \mathbb{R}^{n}$, two times continuously differentiable with respect to $z$ with bounded derivatives, and $g(v, w) \in C^{2}\left(\mathbb{R}^{n} \times \mathbb{R}^{n}\right)$. Then the Newton iteration (4.4) is well-defined and converges quadratically for a starting-value $\bar{s}^{0}$ sufficiently close to the value $\bar{s}^{*}$ associated with an isolated solution of (4.2).

Now we shall formulate an analogous result for the perturbed version of the Newton iteration; the initial value problems (4.2) and (4.5) can in general be solved only with a certain level of accuracy, say $O\left(h^{p}\right)$, on the grid $\Delta_{h}$. We now interpolate (componentwise) the errors from these computations by a piecewise linear function and assume that the continuous function $z_{\bar{s}}(t)+e_{1}(t)$ interpolates the numerical solution of (4.2). Then

$$
\frac{\partial f(t, z)}{\partial z}\left(t, z_{\bar{s}}(t)+e_{1}(t)\right)=\frac{\partial f(t, z)}{\partial z}\left(t, z_{\bar{s}}(t)\right)+E_{1}(t), \quad\left\|E_{1}\right\|=O\left(h^{p}\right),
$$

if $\frac{\partial^{2} f(t, z)}{\partial z^{2}}$ is bounded. Consequently, the approximation for the solution of (4.5) is the numerical solution $\tilde{x}_{h}$ of a perturbed initial value problem

$$
\begin{aligned}
& \tilde{x}^{\prime}(t)=\frac{M(t)}{t} \tilde{x}(t)+\frac{\partial f(t, z)}{\partial z}\left(t, z_{\bar{s}^{m}}(t)\right) \tilde{x}(t)+E_{1}(t) \tilde{x}(t), \quad t \in(0,1], \\
& \tilde{x}(0)=\tilde{E} .
\end{aligned}
$$

Using essentially the same arguments for $x(t)-\tilde{x}(t)$, and making use of the fact that $x(t)$ is bounded (cf. Lemma 4.1), we can show that for a sufficiently small $0<\delta \leq 1$

$$
\|x-\tilde{x}\|_{\delta} \leq \text { const. }\left\|E_{1}\right\|_{\delta}
$$

and classical theory yields

$$
\|x-\tilde{x}\| \leq \text { const. }\left\|E_{1}\right\|
$$

for the whole interval $[0,1]$. Therefore we can conclude that (4.5) can be solved with a level of accuracy $O\left(h^{p}\right)$,

$$
\left\|R_{h}(x)-\tilde{x}_{h}\right\|_{h}=O\left(h^{p}\right) .
$$

If we assume that the first and second derivatives of $g(v, w)$ are bounded as well, we can derive similar expansions for the numerical approximations $A_{\text {num }}(\bar{s})$ for the system matrix $A(\bar{s})$ and the right-hand side of (4.4),

$$
\begin{aligned}
& A_{\text {num }}(\bar{s})=A(\bar{s})+E_{2}(\bar{s}), \quad\left|E_{2}(\bar{s})\right|=O\left(h^{p}\right), \\
& -g\left(z_{\bar{s}}(0), z_{\bar{s}}(1)+e_{1}(1)\right)=-g\left(z_{\bar{s}}(0), z_{\bar{s}}(1)\right)+e_{2}(\bar{s}), \quad\left|e_{2}(\bar{s})\right|=O\left(h^{p}\right) .
\end{aligned}
$$

Thus, the numerical realization of the iteration (4.4) on a fixed grid $\Delta_{h}$ results in a perturbed iteration

$$
\left(A\left(\bar{s}^{m}\right)+E_{2}\left(\bar{s}^{m}\right)\right)\left(\bar{s}^{m+1}-\bar{s}^{m}\right)=-g\left(z_{\bar{s}^{m}}(0), z_{\bar{s}^{m}}(1)\right)+e_{2}\left(\bar{s}^{m}\right),
$$

where $\left|E_{2}\left(\bar{s}^{m}\right)\right|,\left|e_{2}\left(\bar{s}^{m}\right)\right|=O\left(h^{p}\right)$ for sufficiently small $h$ and $\bar{s}^{m}$ sufficiently close to the true solution $\bar{s}^{*}$ of (4.3) associated with an isolated solution of the boundary value problem (4.1).

For a perturbed Newton iteration the following result holds. 
Theorem 4.3. For the solution of the nonlinear operator equation

$$
F(s)=0
$$

we consider the perturbed Newton iteration

$$
\left(F^{\prime}\left(s^{m}\right)+E\left(s^{m}\right)\right)\left(s^{m+1}-s^{m}\right)=-F\left(s^{m}\right)+e\left(s^{m}\right) .
$$

Assume that there exists a solution $s^{*}$ of (4.8) and that (A1) to (A6) hold 4 in a suitably chosen closed ball $B$ with center $s^{*}$ and radius $r$ :

(A1) $\|E(s)\|,\|e(s)\| \leq \varepsilon$, for $\varepsilon$ small and $s \in B$,

(A2) $\|F(s)\| \leq \delta_{0} \forall s \in B$,

(A3) $F(s) \in C^{2}(B)$ and $\left\|F^{\prime \prime}(s)\right\| \leq K \forall s \in B$,

(A4) $F^{\prime}\left(s^{*}\right)$ is nonsingular and $\left\|F^{\prime}\left(s^{*}\right)^{-1}\right\| \leq B_{0}$,

(A5) $B_{0} r<1$,

(A6) $\frac{B_{0} \varepsilon}{1-B_{0} r}<1$.

This implies that

- $\left\|s^{m+1}-s^{*}\right\| \leq$ const. $\left(\left\|s^{m}-s^{*}\right\|^{2}+\varepsilon\right)$,

- for $\left\|s^{m}-s^{*}\right\| \gg \varepsilon$, convergence is quadratic,

- near $s^{*}$ convergence is only linear,

- $s^{m}$ approaches a ball $\mathfrak{E}$ with center $s^{*}$ and radius $r^{*}=O(\varepsilon)$,

- $s^{m}$ is confined to $\mathfrak{E}$ for sufficiently large $m$, but the sequence does not converge to a single point in $\mathfrak{E}$ in general.

Proof. This is a generalization of a standard result (cf. [24]).

Using Banach's Lemma, we can conclude from the assumption (A6) that

$$
\left\|F^{\prime}(s)^{-1} E(s)\right\|<1, \quad s \in B
$$

and hence

$$
\left(F^{\prime}(s)+E(s)\right)^{-1}=F^{\prime}(s)^{-1}+E_{1}(s), \quad\left\|E_{1}\right\| \leq \text { const. } \varepsilon .
$$

Thus, the iteration (4.9) reads

$$
F\left(s^{m}\right)+F^{\prime}\left(s^{m}\right)\left(s^{m+1}-s^{m}\right)=e_{1}\left(s^{m}\right), \quad m=0,1, \ldots,
$$

where

$$
\left\|e_{1}(s)\right\| \leq c_{1} \varepsilon, \quad \forall s \in B
$$

We consider $s$ in a closed ball $\tilde{B}$ around $s^{*}$ with a radius $\tilde{r} \leq r$ such that

$$
\left\|F^{\prime}(s)-F^{\prime}\left(s^{*}\right)\right\| \leq \varepsilon_{1}, \quad s \in \tilde{B},
$$

\footnotetext{
${ }^{4}$ Note that the different operator norms for $F$ and its derivatives are all indiscriminately denoted by $\|\cdot\|$.
} 
and $\varepsilon_{1}<\frac{1}{2 B_{0}}$. If we write $a\left(s^{m}\right):=s^{m+1}$, then for any $s \in \tilde{B}$ and the associated $a(s)$ computed from (4.10) we have

$$
\begin{aligned}
\left\|a(s)-s^{*}\right\| & \leq 2 B_{0}\left(\frac{1}{B_{0}}-\varepsilon_{1}\right)\left\|a(s)-s^{*}\right\| \leq 2 B_{0}\left\|F^{\prime}(s)\left(a(s)-s^{*}\right)\right\| \\
& =2 B_{0}\left\|F^{\prime}(s)\left(a(s)-s^{*}\right)-F^{\prime}(s)(a(s)-s)-F(s)+e_{1}(s)+F\left(s^{*}\right)\right\| \\
& =2 B_{0}\left\|F\left(s^{*}\right)-F(s)-F^{\prime}(s)\left(s-s^{*}\right)+e_{1}(s)\right\| \\
& \leq 2 B_{0}\left(\int_{0}^{1}\left\|F^{\prime}\left(s+\tau\left(s^{*}-s\right)\right)-F^{\prime}(s)\right\| d \tau\left\|s^{*}-s\right\|+\left\|e_{1}(s)\right\|\right) \\
& \leq 2 B_{0}\left(\varepsilon_{1}\left\|s-s^{*}\right\|+c_{1} \varepsilon\right) \\
& <\left\|s-s^{*}\right\|, \quad \text { if } 2 B_{0} c_{1} \varepsilon<\left(1-2 B_{0} \varepsilon_{1}\right)\left\|s-s^{*}\right\|,
\end{aligned}
$$

which means that the iteration (4.10) approaches $s^{*}$ as long as the order of magnitude of the perturbation is small compared to $\left\|s-s^{*}\right\|$.

Since $F^{\prime}$ satisfies a Lipschitz condition with constant $K$, we can conclude from

$$
\left\|F^{\prime}\left(s+\tau\left(s^{*}-s\right)\right)-F^{\prime}(s)\right\| \leq K\left\|s-s^{*}\right\|
$$

that

$$
\left\|a(s)-s^{*}\right\| \leq 2 B_{0} K\left\|s-s^{*}\right\|^{2}+\text { const. } \varepsilon,
$$

which means that when the distance between $s$ and the true solution $s^{*}$ is large compared to the size of the perturbation, quadratic convergence can be observed.

Near $s^{*}$, we interpret (4.10) as a contracting iteration

$$
s^{m+1}=G\left(s^{m}\right) \text {. }
$$

$G$ is contracting with constant $L:=B_{0}^{2} K \delta_{0}$, because the bound $\delta_{0}$ for $F(s)$ can be made arbitrarily small for $s$ near to $s^{*}$. With $\varepsilon_{2}:=\frac{\left\|F^{\prime}(s)^{-1}\right\| c_{1} \varepsilon}{1-L}$ it is easy to see that the following estimates hold:

$$
\begin{aligned}
& \left\|s^{m+1}-s^{*}\right\|<\left\|s^{m}-s^{*}\right\|, \quad \text { for }\left\|s^{m}-s^{*}\right\|>\varepsilon_{2}, \\
& \left\|s^{m}-s^{*}\right\| \leq \varepsilon_{2}, \quad \text { for all sufficiently large } m .
\end{aligned}
$$

For a proof see [31].

Remark. Results similar to the last theorem are numerous in the literature. [13] or 19] for example give proofs of the quadratic convergence of the process if the exact quantities are used. However, the point that the convergence rate drops to 1 near the approximate solution when the numerical quantities are involved is not addressed there. For scalar problems, considerations for a perturbed Newton iteration can be found in [25].

It is clear from the previous considerations that for smooth functions $f$ and $g$ and for a sufficiently small step-size $h$ the assumptions from Theorem 4.3 hold. Thus the following main result holds.

Theorem 4.4. Let $f(t, z)$ be continuous on $[0,1] \times \mathbb{R}^{n}$, and two times continuously differentiable with respect to $z$ with bounded derivatives, and $g(v, w) \in C^{2}\left(\mathbb{R}^{n} \times \mathbb{R}^{n}\right)$, with bounded derivatives. Then the perturbed Newton iteration (4.7) resulting from the numerical solution of (4.1) is well-defined for a suitable initial guess. The iteration converges to an approximation of the true initial value of an isolated solution with order of accuracy $O\left(h^{p}\right)$ provided that $h$ is sufficiently small. Moreover, the convergence of (4.7) is linear near the true solution and quadratic otherwise. 
Having this approximation, the numerical solution $\tilde{z}_{h}$ can be computed in a stable way and the following estimate holds:

$$
\left\|\tilde{z}_{h}-R_{h}(y)\right\|_{h}=O\left(h^{p}\right) .
$$

Proof. Clearly, it only remains to show that the nonlinear problem (4.2) is stable with respect to perturbations in the initial value. This follows from

$$
z_{\bar{s}}(t)=\tilde{E} \bar{s}+t \int_{0}^{1} \tau^{-M(0)}\left(C(\tau t) z_{\bar{s}}(\tau t)+f\left(\tau t, z_{\bar{s}}(\tau t)\right)\right) d \tau
$$

on noting that the right-hand side of the above equation is a contraction for $t \in$ $[0, \delta]$, see [20] or [21].

Remark. In practice, the entries of the Jacobian $A(s)$ are often approximated by finite differences with a suitably small increment $\delta$. This does not change the above considerations substantially. Rather, the same reasoning leads to an estimate

$$
\left\|\tilde{z}_{h}-R_{h}(y)\right\|_{h}=O\left(h^{p}\right)+O(\delta)
$$

for sufficiently small $h$ and $\delta$.

\section{Multiple Shooting}

We begin this section with the analysis of the linear problem (3.1). For a (not necessarily equidistant) mesh

$$
0=\tau_{0}, \tau_{1}, \ldots, \tau_{k}=1
$$

we define the family of initial value problems on the intervals $J_{j}:=\left[\tau_{j}, \tau_{j+1}\right], j=$ $0, \ldots, k-1$,

$$
\begin{aligned}
& \bar{\Phi}_{j}^{\prime}(t)=\frac{M(t)}{t} \bar{\Phi}_{j}(t), \quad t \in J_{j}, \\
& \bar{\Phi}_{j}\left(\tau_{j}\right)=F_{j},
\end{aligned}
$$

where for $j=0, \bar{\Phi}_{0} \in \mathbb{R}^{n \times r}$ and $F_{0}=\tilde{E}$, and for $j=1, \ldots, k-1, \bar{\Phi}_{j} \in \mathbb{R}^{n \times n}$ and $F_{j}$ are nonsingular matrices. For simplicity, we choose $F_{j}=I_{n}, j \geq 1$. The particular solution is defined in an analogous manner,

$$
\begin{aligned}
& v_{j}^{\prime}(t)=\frac{M(t)}{t} v_{j}(t)+f(t), \quad t \in J_{j}, \\
& v_{j}\left(\tau_{j}\right)=0 .
\end{aligned}
$$

Now a vector $\tilde{s}=\left(s_{0}, s_{1}, \ldots, s_{k-1}\right), s_{0} \in \mathbb{R}^{r}, s_{j} \in \mathbb{R}^{n}, j=1, \ldots, k-1$, is determined in such a way that the piecewise function

$$
z_{j}(t):=\bar{\Phi}_{j}(t) s_{j}+v_{j}(t), \quad t \in J_{j}, \quad j=0, \ldots, k-1,
$$

is continuous and coincides with the solution $z(t)$ of (3.2). $\tilde{s}$ can be computed from the matching conditions

$$
z_{j}\left(\tau_{j+1}\right)=z_{j+1}\left(\tau_{j+1}\right), \quad j=0, \ldots, k-2,
$$

and the boundary conditions

$$
B_{a 2} \tilde{E} s_{0}+B_{b 2} \bar{\Phi}_{k-1}(1) s_{k-1}=\beta_{2}-B_{b 2} v_{k-1}(1) .
$$

This is a $((k-1) n+r) \times((k-1) n+r)$ system of linear equations which has a unique solution iff the boundary value problem (3.1) is well-posed. Let us assume that the linear problems (5.1) and (5.2) can be solved in a numerically stable way. Then the 
convergence analysis for multiple shooting reduces to the same considerations as for single shooting, and it follows immediately that the numerical solution of (B.1) obtained by multiple shooting shows the accuracy $O\left(h^{p}\right)$ provided that the initial value problems (5.1) and (5.2) can be solved with the same accuracy on a grid $\Delta_{h}$.

This approach is often called (standard) 5 multiple shooting or parallel shooting, cf. 1], 19. For more sophisticated choices of $F_{j} \in \mathbb{R}^{n \times r}, j \geq 1$, methods like the stabilized march (cf. [29]) can be developed. For singular problems, however, we have to be careful when applying multiple shooting. The reason is the following. The above analysis is valid for a fixed mesh $\tau_{0}, \ldots, \tau_{k}$, where the refinement of the grid for the IVP integrations takes place only in between these mesh points. If the mesh is refined simultaneously with $h \rightarrow 0, \tau_{1}=O(h) \rightarrow 0$, however, a reduction in the convergence order of the method is observed. In general, no order higher than 2 can be expected. For numerical evidence of this phenomenon see Section 6. Consequently, it is necessary to fix (at least) $\tau_{1}>0$ for any stepsize $h$ used in the IVP solver. The stabilized march is an algorithmic measure to make the computations for multiple shooting more efficient, but the result is equivalent to multiple shooting in exact arithmetic. Therefore, we cannot expect the disadvantageous order reduction to recover if this technique is used instead of standard multiple shooting. Indeed, the analysis in [29] reveals that for the stability of the algorithm it is necessary to have the freedom to choose mesh points arbitrarily close to each other. Nonetheless, multiple shooting can still be used to advantage for singular problems if different IVP integrators and mesh selection strategies are to be chosen close to and away from the singularity. An indication that such a strategy may work can be found in 23. Certainly, the stabilized march should also be considered as an interesting alternative for the (regular!) problem on $\left[\tau_{1}, 1\right]$. We would like to stress that multiple shooting with preset mesh points or even single shooting has performed in quite a stable manner for singular problems. This behavior may be explained by the fact that only moderately growing fundamental modes are present in the general solution for a well-posed singular initial value problem.

For the nonlinear problem (4.1) the situation is similar. We define a piecewise problem

$$
\begin{aligned}
& z_{\tilde{s}, j}^{\prime}(t)=\frac{M(t)}{t} z_{\tilde{s}, j}(t)+f\left(t, z_{\tilde{s}, j}(t)\right), \quad t \in J_{j}, \\
& z_{\tilde{s}, j}\left(\tau_{j}\right)=F_{j} s_{j},
\end{aligned}
$$

$j=0, \ldots, k-1$, where $s_{j}$ and $F_{j}$ are chosen as before. In [13] different variants of the stabilized march for nonlinear problems have been proposed. For reasons outlined above we do not discuss these algorithmic measures here, but would certainly consider them as part of an implementation of shooting methods for singular boundary value problems.

The vector $\tilde{s}$ is now determined from the nonlinear system of equations

$$
\tilde{F}(\tilde{s}):=\left(\begin{array}{l}
F_{j+1} s_{j+1}-z_{\tilde{s}, j}\left(\tau_{j+1}\right), j=0, \ldots, k-2 \\
g\left(\tilde{E} s_{0}, z_{\tilde{s}, k-1}(1)\right)
\end{array}\right)=0 .
$$

These equations can be solved using Newton's method. All quantities involved correspond to the quantities occurring in the single shooting procedure 6

\footnotetext{
${ }^{5}$ Except for the choice of $F_{0}=\tilde{E}$ in order to obtain a well-posed initial value problem.

${ }^{6}$ In this case, however, they are defined in a piecewise manner.
} 
Since $s_{1}, \ldots, s_{k-1}$ can again be eliminated from (5.4), multiple shooting for (4.1) is equivalent to single shooting for the same problem defined piecewise on $J_{j}, j=$ $0, \ldots, k-1$. Obviously the solution of (4.1) is isolated iff the solution of the equivalent piecewise problem is. Hence, the Newton iterate $\tilde{s}^{m+1}$ can be uniquely determined from $\tilde{s}^{m}$ if the first component $s_{0}^{m}$ is chosen sufficiently close to the first component of the parameter vector $\tilde{s}^{*}$ associated with an isolated solution. Thus, the Newton iteration is well-defined in a neighborhood of $\tilde{s}^{*}$. Since all other assumptions of Theorem 4.3 can also be shown to hold for multiple shooting in exactly the same manner, we obtain the same convergence result. This means that multiple shooting provides an $O\left(h^{p}\right)$ approximation of an isolated solution of (4.1) if a suitable guess for the starting value is available and an initial value problem solver with convergence order $O\left(h^{p}\right)$ is used.

\section{Numerical Results}

To illustrate the theory, we consider the following nonlinear problem:

$$
\begin{aligned}
& v^{\prime}(t)=\frac{1}{t}\left(\begin{array}{cc}
0 & 1 \\
0 & -1
\end{array}\right) v(t)+t\left(\begin{array}{c}
0 \\
-\frac{2\left(t^{2}+2\right)+8}{\left(t^{2}+2\right)^{2}} v_{1}^{2}(t)+\frac{8 t^{2}}{\left(t^{2}+2\right)^{2}} v_{1}^{3}(t)
\end{array}\right), \quad t \in(0,1], \\
& \left(\begin{array}{ll}
0 & 1 \\
0 & 0
\end{array}\right) v(0)+\left(\begin{array}{ll}
0 & 0 \\
1 & 0
\end{array}\right) v(1)=\left(\begin{array}{c}
0 \\
1 / \ln (3)
\end{array}\right) .
\end{aligned}
$$

Its exact solution reads

$$
v(t)=\left(\frac{1}{\ln \left(t^{2}+2\right)},-\frac{2 t^{2}}{\left(t^{2}+2\right) \ln ^{2}\left(t^{2}+2\right)}\right)^{T} .
$$

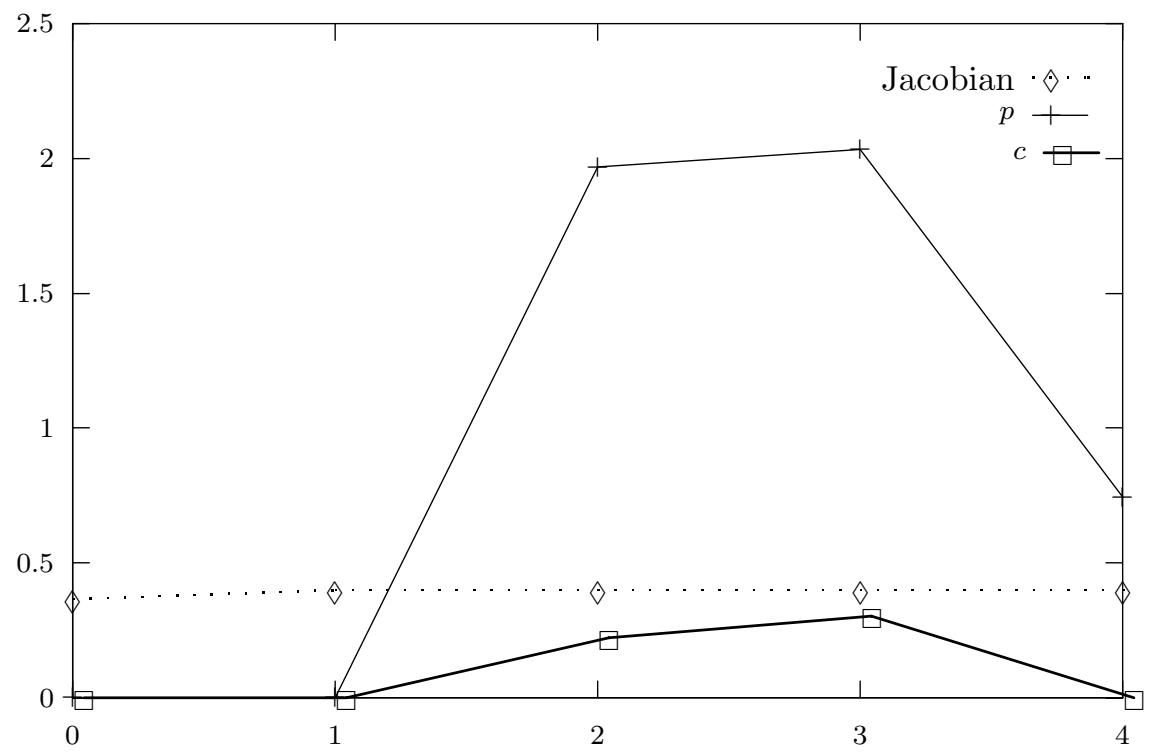

Figure 1. Convergence of Newton for single shooting. 
Table 1. Convergence Order of Single Shooting.

\begin{tabular}{|l||c|c|c||c|c|c|c|}
\hline \multicolumn{1}{|c||}{$h$} & $\delta_{0}$ & $p_{0}$ & $c_{0}$ & $\delta_{[0,1]}$ & $t^{\max }$ & $p_{[0,1]}$ & $c_{[0,1]}$ \\
\hline $1 / 5 \cdot 2^{-1}$ & $6.2 \cdot 10^{-04}$ & 5.989 & $-6.0 \cdot 10^{+02}$ & $3.3 \cdot 10^{-04}$ & 0.10000 & 4.175 & $-4.9 \cdot 10^{+00}$ \\
$1 / 5 \cdot 2^{-2}$ & $9.8 \cdot 10^{-06}$ & 5.065 & $-3.8 \cdot 10^{+01}$ & $1.8 \cdot 10^{-05}$ & 0.15000 & 4.690 & $-2.3 \cdot 10^{+01}$ \\
$1 / 5 \cdot 2^{-3}$ & $2.9 \cdot 10^{-07}$ & 4.971 & $-2.7 \cdot 10^{+01}$ & $7.1 \cdot 10^{-07}$ & 0.20000 & 4.862 & $-4.4 \cdot 10^{+01}$ \\
$1 / 5 \cdot 2^{-4}$ & $9.3 \cdot 10^{-09}$ & 4.976 & $-2.7 \cdot 10^{+01}$ & $2.4 \cdot 10^{-08}$ & 0.26250 & 4.949 & $-6.4 \cdot 10^{+01}$ \\
$1 / 5 \cdot 2^{-5}$ & $2.9 \cdot 10^{-10}$ & 4.985 & $-2.8 \cdot 10^{+01}$ & $7.9 \cdot 10^{-10}$ & 0.25625 & 4.978 & $-7.4 \cdot 10^{+01}$ \\
$1 / 5 \cdot 2^{-6}$ & $9.3 \cdot 10^{-12}$ & 4.992 & $-2.9 \cdot 10^{+01}$ & $2.5 \cdot 10^{-11}$ & 0.25312 & 4.989 & $-7.9 \cdot 10^{+01}$ \\
$1 / 5 \cdot 2^{-7}$ & $2.9 \cdot 10^{-13}$ & 4.995 & $-3.0 \cdot 10^{+01}$ & $7.9 \cdot 10^{-13}$ & 0.25156 & 4.994 & $-8.2 \cdot 10^{+01}$ \\
$1 / 5 \cdot 2^{-8}$ & $9.2 \cdot 10^{-15}$ & 4.997 & $-3.1 \cdot 10^{+01}$ & $2.4 \cdot 10^{-14}$ & 0.25468 & 4.997 & $-8.3 \cdot 10^{+01}$ \\
$1 / 5 \cdot 2^{-9}$ & $2.8 \cdot 10^{-16}$ & 4.998 & $-3.1 \cdot 10^{+01}$ & $7.7 \cdot 10^{-16}$ & 0.25429 & 4.998 & $-8.4 \cdot 10^{+01}$ \\
$1 / 5 \cdot 2^{-10}$ & $9.0 \cdot 10^{-18}$ & 4.999 & $-3.1 \cdot 10^{+01}$ & $2.4 \cdot 10^{-17}$ & 0.25410 & 4.999 & $-8.5 \cdot 10^{+01}$ \\
$1 / 5 \cdot 2^{-11}$ & $2.8 \cdot 10^{-19}$ & & & $7.6 \cdot 10^{-19}$ & 0.25449 & & \\
\hline
\end{tabular}

TABLE 2. Convergence Order of Multiple Shooting, 8 Mesh Points.

\begin{tabular}{|c||c|c|c||c|c|c|c|}
\hline$h$ & $\delta_{0}^{(8)}$ & $p_{0}^{(8)}$ & $c_{0}^{(8)}$ & $\delta_{[0,1]}^{(8)}$ & $t^{\max }$ & $p_{[0,1]}^{(8)}$ & $c_{[0,1]}^{(8)}$ \\
\hline $1 / 5 \cdot 2^{-3}$ & $1.2 \cdot 10^{-06}$ & 3.227 & $-1.7 \cdot 10^{-01}$ & $1.2 \cdot 10^{-06}$ & 0.32500 & 2.978 & $-7.2 \cdot 10^{-02}$ \\
$1 / 5 \cdot 2^{-4}$ & $1.2 \cdot 10^{-07}$ & 4.253 & $-1.6 \cdot 10^{+01}$ & $1.5 \cdot 10^{-07}$ & 0.18750 & 4.237 & $-1.7 \cdot 10^{+01}$ \\
$1 / 5 \cdot 2^{-5}$ & $6.7 \cdot 10^{-09}$ & 4.656 & $-1.2 \cdot 10^{+02}$ & $8.2 \cdot 10^{-09}$ & 0.18750 & 4.642 & $-1.4 \cdot 10^{+02}$ \\
$1 / 5 \cdot 2^{-6}$ & $2.6 \cdot 10^{-10}$ & 4.837 & $-3.5 \cdot 10^{+02}$ & $3.2 \cdot 10^{-10}$ & 0.18125 & 4.824 & $-4.0 \cdot 10^{+02}$ \\
$1 / 5 \cdot 2^{-7}$ & $9.3 \cdot 10^{-12}$ & 4.921 & $-6.0 \cdot 10^{+02}$ & $1.1 \cdot 10^{-11}$ & 0.17968 & 4.917 & $-7.3 \cdot 10^{+02}$ \\
$1 / 5 \cdot 2^{-8}$ & $3.0 \cdot 10^{-13}$ & 4.961 & $-8.0 \cdot 10^{+02}$ & $3.8 \cdot 10^{-13}$ & 0.17812 & 4.959 & $-9.8 \cdot 10^{+02}$ \\
$1 / 5 \cdot 2^{-9}$ & $9.9 \cdot 10^{-15}$ & 4.980 & $-9.4 \cdot 10^{+02}$ & $1.2 \cdot 10^{-14}$ & 0.17773 & 4.979 & $-1.1 \cdot 10^{+03}$ \\
$1 / 5 \cdot 2^{-10}$ & $3.1 \cdot 10^{-16}$ & 4.990 & $-1.0 \cdot 10^{+03}$ & $3.9 \cdot 10^{-16}$ & 0.17773 & 4.990 & $-1.2 \cdot 10^{+03}$ \\
$1 / 5 \cdot 2^{-11}$ & $9.8 \cdot 10^{-18}$ & & & $1.2 \cdot 10^{-17}$ & 0.17744 & & \\
\hline
\end{tabular}

TABLE 3. Convergence Order of Multiple Shooting, $\tau_{j+1}-\tau_{j}=5 h$.

\begin{tabular}{|c||c|c|c||c|c|c|c|}
\hline$h$ & $\delta_{0}^{(5 h)}$ & $p_{0}^{(5 h)}$ & $c_{0}^{(5 h)}$ & $\delta_{[0,1]}^{(5 h)}$ & $t^{\max }$ & $p_{[0,1]}^{(5 h)}$ & $c_{[0,1]}^{(5 h)}$ \\
\hline $1 / 5 \cdot 2^{-2}$ & $3.6 \cdot 10^{-05}$ & 4.926 & $-9.4 \cdot 10^{+01}$ & $3.8 \cdot 10^{-05}$ & 0.15000 & 4.963 & $-1.0 \cdot 10^{+02}$ \\
$1 / 5 \cdot 2^{-3}$ & $1.2 \cdot 10^{-06}$ & 0.828 & $-2.5 \cdot 10^{-05}$ & $1.2 \cdot 10^{-06}$ & 0.32500 & 0.800 & $-2.3 \cdot 10^{-05}$ \\
$1 / 5 \cdot 2^{-4}$ & $6.8 \cdot 10^{-07}$ & 1.939 & $-3.3 \cdot 10^{-03}$ & $7.0 \cdot 10^{-07}$ & 0.15000 & 1.928 & $-3.2 \cdot 10^{-03}$ \\
$1 / 5 \cdot 2^{-5}$ & $1.7 \cdot 10^{-07}$ & 2.048 & $-5.8 \cdot 10^{-03}$ & $1.8 \cdot 10^{-07}$ & 0.07500 & 2.044 & $-5.8 \cdot 10^{-03}$ \\
$1 / 5 \cdot 2^{-6}$ & $4.2 \cdot 10^{-08}$ & 2.047 & $-5.7 \cdot 10^{-03}$ & $4.4 \cdot 10^{-08}$ & 0.03750 & 2.044 & $-5.9 \cdot 10^{-03}$ \\
$1 / 5 \cdot 2^{-7}$ & $1.0 \cdot 10^{-08}$ & 2.030 & $-5.1 \cdot 10^{-03}$ & $1.0 \cdot 10^{-08}$ & 0.01875 & 2.029 & $-5.3 \cdot 10^{-03}$ \\
$1 / 5 \cdot 2^{-8}$ & $2.5 \cdot 10^{-09}$ & 2.017 & $-4.7 \cdot 10^{-03}$ & $2.6 \cdot 10^{-09}$ & 0.00937 & 2.016 & $-4.8 \cdot 10^{-03}$ \\
$1 / 5 \cdot 2^{-9}$ & $6.2 \cdot 10^{-10}$ & 2.009 & $-4.4 \cdot 10^{-03}$ & $6.5 \cdot 10^{-10}$ & 0.00468 & 2.008 & $-4.5 \cdot 10^{-03}$ \\
$1 / 5 \cdot 2^{-10}$ & $1.5 \cdot 10^{-10}$ & & & $1.6 \cdot 10^{-10}$ & 0.00234 & & \\
\hline
\end{tabular}

All computations were carried out on a Silicon Graphics Power Challenge XL R10000 with operating system IRIX V.4. Calculations were performed in quadruple precision, which on this system implies the machine accuracy EPS $\approx 6.16 \cdot 10^{-33}$.

Table 1 shows the convergence results for single shooting, Table 2 for multiple shooting with 8 mesh points $\tau_{j}$. Moreover, Table 3 shows an example where multiple shooting fails when the mesh is refined simultaneously with the step-size for the IVP integration. The tables list (for each step-size $h$ ) the error in the numerically computed initial value $7 \delta_{0}$, the convergence order, and the error constant, denoted by $p_{0}, c_{0}$, respectively. Here, we assume a relation $\delta_{0}=c_{0} h^{p_{0}}$ to hold. The subscript

\footnotetext{
${ }^{7}$ For multiple shooting, we use the same notation for the starting values in every point $\tau_{j}, j=$ $0, \ldots, k-1$.
} 
$[0,1]$ is related to the same quantities computed for the maximal error over the entire interval. $t^{\max }$ indicates the point where this maximum is assumed. The underlying IVP solver has an order of convergence of $O\left(h^{5}\right)$. As predicted by the theory, we observe the same convergence order for the shooting procedure, cf. Tables 1 and 2 A reduction of the convergence order down to order 2 is clearly visible in case of the simultaneous grid refinement, $\tau_{j+1}-\tau_{j}=5 h, j=0, \ldots, k-1$, see Table 3 ,

Figure 1 shows (the numbers of the iteration steps are displayed on the horizontal axis) the empirical convergence order of Newton's method for the computation of the approximate solution of 4.30). Here, a finite difference approximation for the Jacobian with an increment $\delta=\sqrt{\text { EPS }}$ was used, see the remark on page 300. The order of convergence is denoted by $p$ and the error constant computed from the errors of three consecutive Newton iterates by $c$, that is, $\left|\bar{s}^{m+1}-\bar{s}^{m}\right|=$ $c\left|\bar{s}^{m}-\bar{s}^{m-1}\right|^{p}$. Moreover, the modulus of the Jacobian $A_{\text {num }}\left(\bar{s}^{m}\right)$ for every iteration step is given. As predicted, the scheme (4.7) converges quadratically except for the last step, where convergence drops to linear because the numerical solution is already close to the true one.

We refer to [2] for more experiments and for investigations of further aspects related to the implementation of the shooting method.

\section{REFERENCES}

1. U. Ascher, R. Mattheij, and R. Russell, Numerical Solution of Boundary Value Problems for Ordinary Differential Equations, Prentice-Hall, Englewood Cliffs, NJ, 1988. MR 96f:65075 (reprint)

2. W. Auzinger, O. Koch, P. Kofler, And E. Weinmüller, The application of shooting to singular boundary value problems, Techn. Rep. Nr. 126/99, Inst. for Appl. Math. and Numer. Anal., Vienna Univ. of Technology, Austria, 1999. Available at http://fsmat.at/ othmar/research.html.

3. E. Badralexe And A. Freeman, Eigenvalue equation for a general periodic potential and its multipole expansion solution, Phys. Rev. B, 37 (1988), pp. 1067-1084.

4. L. Bauer, E. Reiss, And H. Keller, Axisymmetric buckling of hollow spheres and hemispheres, Comm. Pure Appl. Math., 23 (1970), pp. 529-568. MR 43:4335

5. T. CARR AND T. ERNEUx, Understanding the bifurcation to traveling waves in a class- $b$ laser using a degenerate Ginzburg-Landau equation, Phys. Rev. A, 50 (1994), pp. 4219-4227.

6. C. Chan AND Y. Hon, A constructive solution for a generalized Thomas-Fermi theory of ionized atoms, Quart. Appl. Math., 45 (1987), pp. 591-599. MR 88j:34034

7. M. Drmota, R. Scheidl, H. Troger, and E. Weinmüller, On the imperfection sensitivity of complete spherical shells, Comp. Mech., 2 (1987), pp. 63-74.

8. R. FAZIO, A novel approach to the numerical solution of boundary value problems on infinite intervals, SIAM J. Numer. Anal., 33 (1996), pp. 1473-1483. MR 97e:65069

9. R. Frank, The method of Iterated Defect Correction and its application to two-point boundary value problems, Part I, Numer. Math., 25 (1976), pp. 409-419. MR 56:4180a

10. value problems, Part II, Numer. Math., 27 (1977), pp. 407-420. MR 56:4180b

11. R. Frank AND C. ÜBerhuber, Iterated Defect Correction for Runge-Kutta methods, Techn. Rep. Nr. 14/75, Inst. for Appl. Math. and Numer. Anal., Vienna Univ. of Technolgy, Austria, 1975.

12. F. Frommlet And E. Weinmüller, Asymptotic error expansions for singular boundary value problems, Math. Models Methods Appl. Sci., 11 (2001), pp. 71-85. CMP 2001:09

13. M. Hermann AND D. KAISER, Shooting methods for two-point BVPs with partially separated endconditions, ZAMM, 75 (1995), pp. 651-668. MR 96i:65061

14. F. DE Hoog AND R. WeIss, Difference methods for boundary value problems with a singularity of the first kind, SIAM J. Numer. Anal., 13 (1976), pp. 775-813. MR 55:13799

15. - The application of linear multistep methods to singular initial value problems, Math. Comp., 31 (1977), pp. 676-690. 
16. Collocation methods for singular boundary value problems, SIAM J. Numer. Anal., 15 (1978), pp. 198-217. MR 57:8041

17. - The application of Runge-Kutta schemes to singular initial value problems, Math. Comp., 44 (1985), pp. 93-103. MR 86h:65100

18. H. B. Keller, Numerical Methods for Two-Point Boundary-Value Problems, Blaisdell Publishing Company, Waltham, Mass., 1968. MR 37:6038

19. _ Numerical Solution of Two Point Boundary Value Problems, no. 24 in Regional Conference Series in Applied Mathematics, SIAM, Philadelphia, 1976. MR 55:6868

20. O. Koch, P. Kofler, And E. Weinmüller, Analysis of singular initial and terminal value problems, Techn. Rep. Nr. 125/99, Inst. for Appl. Math. and Numer. Anal., Vienna Univ. of Technology, Austria, 1999. Available at http://fsmat.at/ othmar/research.html.

21. lems, Appl. Num. Math., 34 (2000), pp. 231-252. MR 2001f:65079

22. O. Koch And E. WeinmüLler, Iterated Defect Correction for the solution of singular initial value problems. To appear in SIAM J. Numer. Anal.

23. P. Koflen, Theorie und numerische Lösung singulärer Anfangswertprobleme gewöhnlicher Differentialgleichungen mit der Singularität erster Art, Ph. D. Thesis, Inst. for Appl. Math. and Numer. Anal., Vienna Univ. of Technology, Austria, 1998.

24. P. Kosmol, Methoden zur numerischen Behandlung nichtlinearer Gleichungen und Optimierungsaufgaben, Teubner, Stuttgart, 1989.

25. P. LANCASTER, Error analysis for the Newton-Raphson method, Numer. Math., 9 (1966), pp. 55-68. MR 35:1208

26. X. Liu, A note on the Sturmian Theorem for singular boundary value problems, J. Math. Anal. Appl., 237 (1999), pp. 393-403. MR 2000f:34049

27. R. MÄrz AND E. Weinmüller, Solvability of boundary value problems for systems of singular differential-algebraic equations, SIAM J. Math. Anal., 24 (1993), pp. 200-215.

28. G. Moore, Computation and parametrization of periodic and connecting orbits, IMA J. Numer. Anal., 15 (1995), pp. 245-263. MR 96a:34087

29. M. R. Osborne, The stabilized march is stable, SIAM J. Numer. Anal., 16 (1979), pp. 923933. MR 81e:65047

30. S. Parter, M. Stein, And P. Stein, On the multiplicity of solutions of a differential equation arising in chemical reactor theory, Techn. Rep. Nr. 194, Dept. Computer Sciences, Univ. of Wisconsin, 1973 = Studies in Appl. Math., 54 (1975), pp. 293-314. MR 56:10026

31. H. J. SteTteR, Numerik für Informatiker: computergerechte numerische Verfahren, Oldenbourg, Wien-München, 1976. MR 54:1531

32. R. WeISS, The convergence of shooting methods, BIT, 13 (1973), pp. 470-475. MR 48:12856

33. H. Werner And H. ARndt, Gewöhnliche Differentialgleichungen: eine Einführung in Theorie und Praxis, Springer-Verlag, Berlin-Heidelberg, 1986. MR 88b:34002

Department of Applied Mathematics and Numerical Analysis, University of Technology Vienna, Wiedner Hauptstrasse 8-10, A-1040 Vienna, Austria

E-mail address: othmar@fsmat.at

Department of Applied Mathematics and Numerical Analysis, University of Technology Vienna, Wiedner Hauptstrasse 8-10, A-1040 Vienna, Austria

E-mail address: e.weinmueller@tuwien.ac.at 